\title{
The Influence of Social Media on Contemporary Subcultural Groups in China: The Case of BL Fandom*
}

\begin{abstract}
CHEN Min
China Youth University of Political Studies, Beijing, China

Social media has become the infrastructure of contemporary virtual life and greatly influenced the landscapes of the modern culture. By tracking the development of a local subculture in China-BL fandom and analyzing BL fandom on social media, this research argues that social media empowers the subcultural groups and makes the cultural expression of BL fans heard. Meanwhile, the social relationship of online subcultural groups shows the tendency of decentralization and conglomeration, which is typical of social network services. In the interaction between fujoshies through social media, the subcultural groups also develop a collective identity resistant against the mainstream society. The rise of social media leads the contemporary subcultures to a new stage.
\end{abstract}

Keywords: social media, subcultural groups, BL fandom

\section{Introduction}

Social media refers to Web 2.0 Internet-based applications that allow people to create and exchange content using digital network technologies (Boyd \& Ellison, 2008; Kaplan \& Haenlein, 2010). According to 2012 CCIC report, the number of social media users in China reached 275 million. They mainly engage in the social applications such as QQ, blog, microblog, wechat, and other social network sites such as Post Bar of Baidu, Douban, and Renren. The boom of social media in China in the recent decade makes a lasting influence on Chinese society. Since youth are the main force of virtual community in China, social media affects every aspects of Chinese youth life, including their subcultures. The relationship between the social media and the contemporary Chinese subcultures has been increasingly researched and measured by scholars at home and abroad.

Chinese youth subcultures emerged in 1980s and developed fast in the new millennium. Its fast development attracted the attention of Chinese scholars. Many scholars consider social media a positive factor for Chinese youth subcultures. MA Zhong-hong (2010), when discussing the relationship between the new media and youth subcultures, argued that the new media brought about changes to the style, content, and forms of youth subcultures, enabled the subculture to have more immediate impacts on the mainstream culture, and therefore put subcultures at a more advantageous position. Hsin Yen Yang (2010), on the other hand, discussed the effects of social media on Japanese drama fans. He noted that the social media drew more attention of these fans to social politics and produced potentials of social transformation. CAI Qi and HUANG Yao-ying (2011)

\footnotetext{
* Acknowledgements: The research is supported by the research project of China Youth University of Political Studies "The Influence of Social Media on Contemporary Youth Subcultures" (Serial No.189070823).

CHEN Min, lecturer, M.A., English Department, China Youth University of Political Studies.
} 
investigated two Chinese social websites, Douban and Renren. They maintained that social media itself was a cradle of youth subcultures in which subcultures grow and spread. On the whole, the scholars hold the social media positively. However, there is little comprehensive research, if not none, on how social media works in a particular Chinese subculture. This research is attempted to analyze a particular Chinese subculture-BL fandom in China and explore the impact of social media on it so as to observe the influence of social media on Chinese youth subcultures more thoroughly.

\section{BL Fandom in China}

BL stands for boys' love. As a sub-genre of shoujo manga that originated and developed in 1970s' Japan, Japanese BL manga and fiction were mainly about the love stories between beautiful boys. It is noticed that BL manga and fiction were produced by female authors and targeted for young female readers. These female fans of BL manga and fiction mocked themselves and labeled themselves as fujoshi, which literally means a rotten girl. This exclusive female authorship and readership became a noticeable feature of BL fandom wherever it spreads.

With its increasing popularity, Japanese BL manga and fiction spread its influence across the national borders. In Southeast Asia and America, local BL fandom also emerged. With the development of the Internet, fujoshies exchanged and spread considerable BL works online. In 2006, the search results of English web pages related to BL culture amounted to 3,740,000, of Spanish web pages 639,000, and Italian pages 181,000. Andrea Wood indicated that the transnational circulation of BL manga had developed a global and resistant counterpublic that is both subversive and queer in nature.

In early1990s, BL manga and fiction entered China and brought about the popularity of BL culture. The Chinese fujoshies began to write local BL fiction in late 1990s. With the development of the Internet, online BL fandom was established in mainland China. The first Chinese BL website Sunsun Academy (see http://sunsunplus.51.net) came into use in 1998. Later on, more and more BL fansites were set up. These websites kept track of the latest BL trends, published original BL stories produced by Chinese BL authors, providing the BL fans an access to BL culture and a virtual community for communication of BL fans. Besides these specialized BL fansites, some literary websites such as Jing Jiang Literary City and Lian Cheng Literary Network also maintained sections for BL fiction. Based on the author's investigation, the search results of Chinese web pages related to BL fandom reached 100,000,000 by 2016. Chinese BL fandom grew steadily as an important part of the local youth subcultures.

\section{The Influence of Social Media on BL Fandom in China}

In the development of Chinese BL fandom, social media plays an important role. As the theme of $\mathrm{BL}$ fiction is related to the romantic relationship of beautiful boys and typical BL fiction is erotically charged, the BL fiction is not readily accepted by Chinese mainstream. Therefore, the Internet becomes the major channel, if not the only, for production and circulation of BL fictions. On the Internet, the BL fictions are shared and circulated mainly on fansites and general social websites.

Take Lucifer Club (see www.lucifer-club.com) as an example. Launched in 1999, it claimed to be the biggest BL fansite in China. By February 2016, a total of 72,170 stories have been posted in Lucifer Club, majority of which were original BL stories published for the first time except 297 romantic narratives. The Lucifer Club offers the services such as Anthology, Chart, Forum, and Chat Room for the BL fans. Anthology 
section shares all the works published on Lucifer and classifies them into different types. The Chart shows the list of BL works according to the rate of clicking, update time, favorites of the users and ratings. In Forum, BL writers promote their works and the fans exchange their reviews and comments about the BL stories. The Chat Room offers the space for BL writers and fans to communicate online. In the early days of BL fandom, Lucifer Club greatly promoted the production and circulation of BL stories. Many famous BL writers in China such as FengNong and Apple gain their popularity in BL fans through Lucifer Club.

Another important BL forum is the Post Bar of Baidu. It was launched in 2003 and claimed as the largest online community in China. Since its birth, sub-communities of Baidu Post Bar have mushroomed, among which the most popular were BL Bar, Yaoi Bar, the Events Record Bar. The first two are comprehensive BL fansites while the latter one is dedicated to sharing of BL fictions. By March 2016, the number of BL Bar and Yaoi Bar users reached 1,000,000 and 2,000,000, and the posts amounted to 20,000,000 and 8,000,000 respectively. The fujoshies usually share the BL stories, manga and games, and discuss the BL stories. The green hands of BL fiction also take the bar as a lab for their BL writing.

The various social websites provide the immense virtual space for BL authors and fans who cannot otherwise participate in BL culture in reality. They share BL fiction and exchange their comments. The BL fan community, which used to be a marginalized subcultural group, is therefore empowered to articulate their suppressed discourse and came into view of the public. The flourishing social websites is the solid base for the enlarged voiced of China's fujoshies community and the battlefield for them to struggle for power in Chinese cultural sphere.

Meanwhile, the interaction of fujoshies community is also facilitated by social media. The online interaction on social media is different from the offline interaction of subcultural groups. In off line interaction, the group members have to coexist in physical space to interact and therefore realize their communication. It is difficult for them to interact with each other if they live in different places and have different social backgrounds. But on social websites, the subcultural group members do not have to communicate face to face. Social media provides a common virtual space for them to coexist so their networks of interaction are expanded and communication is enhanced. In addition, the organization of the social media is decentralized. The members in online community serve as an independent node in the information network, breaking the traditional vertical relational structure and revealing the features of horizontal relations. The decentralization of interpersonal relationship promotes the development of flat and equal relationship between subcultural group members.

In the case of BL fandom, fujoshies participate in the BL community by interacting with the BL authors and other fujoshies. On the social websites, fujoshies interact with the BL authors by writing online comments. As the BL stories usually appear as serials, the fujoshi readers can post their comments after the each serial part. Therefore, the commenting posts run through the whole serials and can be read both by authors and other readers. Whether long or short, positive or negative, these posts help the BL authors to lessen their loneliness, feel the connection with the BL community, and therefore encourage their writing. Take the comments on Dear Baby, a Chinese BL story posted on Lucifer Club in 2006 as example. Written by Bei, an amateur BL author, it describes the life of a young man, Hanyuan and his adopted child who is 14 years younger than he. The story has topped the Favorite List of Lucifer Club and the posts following the story amounted to 11,400. Some analyzed the writing techniques, predicted the ending of the story; some plead the author to post the story more quickly while others challenge the logic of the writing and even offer suggestions for the author. The author 
also posted her response to the fans, explaining her writing and expressing her feelings. She even wrote a postscript to describe her writing process and thank a list of warm-hearted fujoshies, referring them as Your Grace. The multiple voices found in interaction between BL authors and fujoshies bear a decentralized relation network in the community and help them get involved more in BL culture.

On the other hand, the development of social media also allows the conglomeration of the subcultural groups within the community. Blogs and microblogs are such social media. Compared with the group-oriented sites and forums, these web services are more individualized and operate on a one-to-many manner. The users can share their own resources and links to other resources. At the same time, the readers are allowed to comment and see their interests. Many BL authors take the advantage of new social media and launch their own microblogs. FengNong, for example, registered an account of Sina Weibo, which was the largest microblog service in China. Her followers on Weibo reached 450,000. Her Weibo is divided into three sections: the Homepage, the Album, and the Works List. On the Homepage, she shares her daily life activities and her opinions on various issues. For example, she uploaded the picture of her study. In March 2016, she wrote a remark about the TV Series Rules and challenged the prohibition of homosexuality in the rules: "Hasn't homosexuality been officially confirmed neither a disease nor a crime?" Her comment excited a lot of response from the followers. From 2010 to 2015, she wrote more than 7,000 Weibos, which allow her BL fans to learn her and interact with her frequently. In her Album, FengNong shared many comic pictures of her BL novel Phoenix in the Sky. In the Works List, she listed all of her works, including the links of the introduction and the purchase. The followers can learn her novels and comment on them. FengNong's Weibo exposes herself and her works effectively to the fans and provides a convenient channel for the BL fans to communicate with her. As the opinion leaders of BL community, BL authors attracted many followers through social media and reinforced their position of advantage in the relational structure of BL community.

Social media helps attract fujoshies to the BL authors of subcultural groups. It also allows conglomeration of more subgroups in the BL fan community. In the virtual BL community of social media, the members can also join different groups based on their interested topics. For example, BL Bar has more than 200 discussion subgroups, which recruit BL fans with more specific common interest in things such as comics, singing, ancient BL stories, and original BL stories. The conglomeration of subgroups creates closer ties between community members.

In providing the virtual cultural space for the interaction of subcultural members, social media also helps create the collective identity among the members of subcultural groups. Collective identity is related to question of "who we are". In the Post Bar of Baidu, one cannot join the community unless he completes a process of registration. At the same time, the BL community is managed by various moderators and administrators at different levels and has clear rules for the format of posts, the range of topics, and the use limit of community resources. If a member violates the rules of the community, he will be forbidden to post or be deprived of membership. The rules of the community ensure the operation of the BL social websites and imply the members in the communal should behave within known constraints of forum, therefore creating the collective consciousness. Meanwhile, obtaining a senior membership of the community often takes a longer period of time. For example, the members can gain a higher status only by writing posts actively and signing in the bar every day

Another important BL community, the Lucifer Club does not only have similar regulations for its members' behaviors, but also have higher requirement for admission to the club. Only when the applicant 
answers five difficult questions related to BL fictions correctly can he become a member of Lucifer Club. Excluding outsiders in that way shows that the BL community is highly aware of its difference from other cultures. By building up a boundary between the group members and outsiders, it establishes a highly committed and insular subcultural community and a strong collective identity.

The collective identity is not only built through the rules and regulations; it also comes into being in the open and free discussions of BL fans on the sensitive topics in the forum. On Lucifer Club, for example, topics range from their BL reading experience to daily life experience. The hot BL topics include their favorite $\mathrm{BL}$ authors, stories, and the characters, how they become BL fans and the reaction of their parents when learning they are BL fans, etc. For example, when the BL fans discussed the reaction of their parents when discovering they read BL stories, most of BL fans admitted the parents' tolerance and ridiculed their attempt to prevent the children from reading BL stories. The discussion of shared experience of confronting mainstream values creates emotional ties between them. Daily life topics include their backgrounds, their location, their majors, and their sexual tendency in reality, etc. Due to the absence of physical existence BL fans do not have to worry about disclosure of their personal information or feel embarrassed. In discussing the forbidden topics in social websites freely, the BL fans develop their resistant awareness against the status-quo and build a collective identity of BL community.

\section{Conclusion}

Social media has become important infrastructure of contemporary virtual life and greatly influenced the landscapes of the online subcultures. Based on social media, subcultural groups are empowered to establish their virtual community and make their cultural expression heard. Meanwhile, the social relationship of online subcultural groups shows the tendency of decentralization and conglomeration. In the interaction within community through social media, the subcultural groups also develop a collective identity resistant against the mainstream society. The social media empowers the subcultural groups and develops the power relationship both within and outside the subcultural community.

\section{References}

Boyd, D., \& Ellison, N. B. (2008). Social network sites: Definition, history, and scholarship. Journal of Computer-Mediated Communication, 13(1), 210-230.

CAI, Q., \& HUANG, Y. Y. (2011). The subcultural communication of SNS virtual community. Contemporary Communication, 1, 14-16.

Kaplan, A. M., \& Haenlein, M. (2010). Users of the world, unite! The challenges and opportunities of social media. Business Horizons, 53(1), 59-68.

MA, Z. H. (2010). New media and the turn of youth subcultures. Journal of Literary and Art Research, 10, 104-112.

Wood, A. (2006). "Straight" women, queer texts: Boy-love manga and the rise of a global counterpublic. Women's Studies Quarterly, 34, 396-397.

YANG, H. Y. (2010). Re-interpreting Japanomania: Transnational media, national identity and the restyling of politics in Taiwan (Ph.D. dissertation, University of Iowa). 